\title{
Oncological outcome, complications, lower urinary tract symptoms, and health-related quality of life after low-dose-rate salvage brachytherapy for recurrent prostate cancer following primary radiotherapy: a report of 8 cases
}

\author{
Makito Miyake, MD!, Nobumichi Tanaka, MD!, Isao Asakawa, MD², Shunta Hori, MD', Yosuke Morizawa, MD!', \\ Yasushi Nakai, MD', Satoshi Anai, MD', Kazumasa Torimoto, MD', Katsuya Aoki, MD', Nagaaki Marugami, MD³, \\ Masatoshi Hasegawa, MD², Tomomi Fujii, MD4, Noboru Konishi, MD4, Kiyohide Fujimoto, MD' \\ 'Department of Urology, 2Department of Radiation Oncology, ${ }^{3}$ Department of Radiology, ${ }^{4}$ Department of Pathology, Nara Medical University, \\ Nara, Japan
}

\begin{abstract}
Purpose: We evaluated our experience with low-dose-rate salvage brachytherapy for local recurrence after primary prostate radiotherapy, and described the changes in lower urinary tract symptoms and health-related quality of life.

Material and methods: Between 2011 and 2016, eight men with local recurrence after primary prostate radiotherapy underwent iodine-125 salvage brachytherapy with a prescribed dose of 110 or 145 Gy. Recurrence-free survival was evaluated with a post-treatment prostate-specific antigen profile. The toxicity and changes in lower urinary tract symptoms and health-related quality of life during the follow-up were evaluated on the Common Terminology Criteria for Adverse Events version 4.0, International Prostate Symptom Score, Short Form-8, and Expanded Prostate Cancer Index Composite, respectively.

Results: The median follow-up was 12.2 months (range, 8.3-71.9) after salvage brachytherapy. Of all eight patients, two $(25 \%)$ experienced treatment failure, one of whom developed left seminal vesicle recurrence 36 months after salvage brachytherapy for the right seminal vesicle recurrence, while the other developed bone metastases after 6 months. The International Prostate Symptom Scores peaked at 3 months, and returned to baseline by 6 months. The scores of all domains of health-related quality of life remained unchanged during the 12-month follow-up after salvage brachytherapy. Early grade $\leq 2$ genitourinary toxicity was observed in five patients (63\%), and late grade 2 gastrointestinal toxicity in one patient $(13 \%)$ having persistent diarrhea. No patient required intermittent catheterization and no grade 3 or greater toxicity occurred during follow-up.

Conclusions: The present study is our experiment of eight patients undergoing salvage brachytherapy, suggesting that this modality is noninvasive, safe, and an effective salvage local treatment in selected patients. To our knowledge, this is the first study to evaluate lower urinary tract symptoms and health-related quality of life in the post-treatment period in prostate cancer patients.
\end{abstract}

Key words: LUTS, prostate cancer, QOL, salvage brachytherapy.

\section{Purpose}

In the clinical management of prostate cancer $(\mathrm{PCa})$, the elevation of serum prostate-specific antigen (PSA) levels after primary radical treatment is the consequence of a local recurrence or a distant metastasis, in which androgen deprivation therapy (ADT) is considered as the first- line therapy [1]. However, a substantial proportion of PSA failure is associated with localized recurrent tumors, not with metastatic disease. The management of patients with local recurrent PCa after primary local radiotherapy has been controversial $[2,3,4]$. Isolated local recurrence in the prostate or seminal vesicles (SVs) may benefit from local salvage therapy. The National Comprehensive Cancer

Address for correspondence: Nobumichi Tanaka, MD, Department of Urology, Nara Medical University, Received: 25.06 .2017 840 Shijo-cho, Nara 634-8522, Japan, phone: +81 744223051 (ext. 2338), fax: +81 744229282, 
Network (NCCN) guideline version 2017 endorses the use of salvage local therapy, such as salvage prostatectomy with lymph node dissection, high-dose-rate (HDR) or low-dose-rate (LDR) salvage brachytherapy (SBT), or salvage cryotherapy in selected men with biopsy-proven local recurrences [5]. In addition, high-intensity focused ultrasound (HIFU) has been reported to be an effective focal modality with acceptable clinical outcomes in the salvage setting [6].

Low-dose-rate brachytherapy (LDR-BT) using iodine-125 $\left({ }^{125} \mathrm{I}\right)$ seed implantation is currently one of the standard treatments for localized PCa [7]. Recent publications have shown that LDR-SBT can be applied as potentially curative local approaches after primary radical radiotherapy $[8,9,10,11,12,13,14,15,16]$. There are clinical concerns that LDR-SBT may cause more frequent incidences of adverse events and severe deterioration in lower urinary tract symptoms (LUTS) and quality of life (QOL) than when used as a primary treatment. However, data regarding the post-reimplantation changes in LUTS and QOL are limited. Herein, we report a single center cohort experience of LDR-SBT with clinical outcome including oncological control, complications, and changes in LUTS and health-related QOL (HR-QOL).

\section{Material and methods}

\section{Patients and data collection}

Between April 2004 and June 2016, 1,008 consecutive patients underwent ${ }^{125} \mathrm{I}$ brachytherapy for PCa at the Nara Medical University, Nara, Japan. Out of 1,008 patients, eight underwent ${ }^{125}$ I seed implantation as a salvage setting after primary radiotherapy. The individual clinicopathologic characteristics, the primary radiotherapy, and PSA nadir after the radiotherapy are shown in Table 1 . Two pathologists with expertise in PCa diagnosis reviewed the Gleason scores (GS) of all biopsy specimens. Tumor stages were identified according to the 2002 Union for International Cancer Control classification. Patients were stratified according to the D' Amico risk classification [17]. Five were initially treated with LDR-BT, two were treated with intensity-modulated radiotherapy (IMRT), and the remaining one was treated with proton beam radiotherapy. Prostate T2-weighted and diffusion-weighted magnetic resonance imaging (MRI), chest/abdomen/pelvis computed tomography (CT), and bone scintigraphy were routinely performed. All eight patients underwent template transperineal saturation biopsy of the prostate and bilateral seminal vesicle (optional, six cores) to confirm local recurrence pathologically. The number of biopsy cores of the prostate depended on the prostate volume. The preSBT PSA doubling time (PSADT) between the post-radiation PSA nadir and the SBT was calculated using at least two PSA measurements with a 3-month interval and log calculations from the website of the Memorial Sloan Kettering Cancer Center [18].

The methods and procedures for this study were approved by the Ethics Committee of the Nara Medical University, and all participants provided informed consent before treatment and testing.

\section{Procedure of the low-dose-rate salvage brachytherapy}

We performed the ultrasound-guided implantation procedure with preplanning and real-time planning using a VariSeed 7.2 planning system (Varian Medical Systems, Palo Alto, CA, USA), and ${ }^{125}$ I radioactive seeds (OncoSeed, GE Healthcare, Medi-Physics Inc., Arlington Heights, IL, USA) using Mick's applicator as previously described $[19,20]$. A radiologist with expertise of urogenital diseases diagnosed prostate MRI based on quantitative T2-weighted imaging, diffusion weighted imaging (DWI), and dynamic contrast enhanced MRI. During SBT, the clinical target volume (CTV), which was equal to the planning target volume, was set to recurrent regions, which were detected by needle biopsy and/or prostatic MRI. SBT was conducted to minimize genitourinary (GU) and gastrointestinal (GI) toxicity by positioning seeds away from the urethra and rectal wall, respectively. The dose was prescribed to the $100 \%$ isodose line covering the CTV. Prostatic MRI showed no abnormalities outside the CTV. An experienced radiation oncologist performed a CT scan about 1 month after implantation to obtain the post-implant dosimetric parameters.

Table 1. Initial diagnosis and primary treatment

\begin{tabular}{|c|c|c|c|c|c|c|c|}
\hline $\begin{array}{l}\text { Patient } \\
\text { No. }\end{array}$ & $\begin{array}{c}\text { Age at } \\
\text { diagnosis }\end{array}$ & $\begin{array}{l}\text { Initial PSA } \\
(\mathrm{ng} / \mathrm{ml})\end{array}$ & $\begin{array}{l}\text { Biopsy positive/total } \\
\text { (Gleason score) }\end{array}$ & T stage & $\begin{array}{l}\text { D’Amico risk } \\
\text { stratification }\end{array}$ & $\begin{array}{l}\text { Initial } \\
\text { radiotherapy }\end{array}$ & $\begin{array}{l}\text { PSA nadir } \\
(\mathrm{ng} / \mathrm{ml})\end{array}$ \\
\hline 1 & 65 & 5.5 & $4 / 8(3+3)$ & $\mathrm{T} 1 \mathrm{c}$ & Low & BT & 0.4 \\
\hline 2 & 76 & 27.0 & $2 / 37(3+3)$ & $\mathrm{T} 2 \mathrm{a}$ & High & IMRT & 1.7 \\
\hline 3 & 57 & 9.6 & $3 / 10(4+3)$ & $\mathrm{T} 1 \mathrm{c}$ & Intermediate & BT & 0.6 \\
\hline 4 & 57 & 6.7 & $1 / 8(3+3)$ & $\mathrm{T} 1 \mathrm{c}$ & Low & BT & 0.5 \\
\hline 5 & 64 & 8.7 & $3 / 12(3+4)$ & $\mathrm{T} 2 \mathrm{a}$ & Intermediate & Proton beam & $<0.1$ \\
\hline 6 & 50 & 11.0 & $1 / 8(4+4)$ & $\mathrm{T} 2 \mathrm{~b}$ & High & IMRT & 0.1 \\
\hline 7 & 61 & 7.5 & $1 / 10(3+3)$ & $\mathrm{T} 1 \mathrm{C}$ & Low & BT & 0.7 \\
\hline 8 & 66 & 6.8 & $5 / 12(3+4)$ & $\mathrm{T} 2 \mathrm{a}$ & Intermediate & BT & 0.3 \\
\hline
\end{tabular}

PSA - prostate-specific antigen, BT - brachytherapy, IMRT - intensity-modulated radiotherapy 


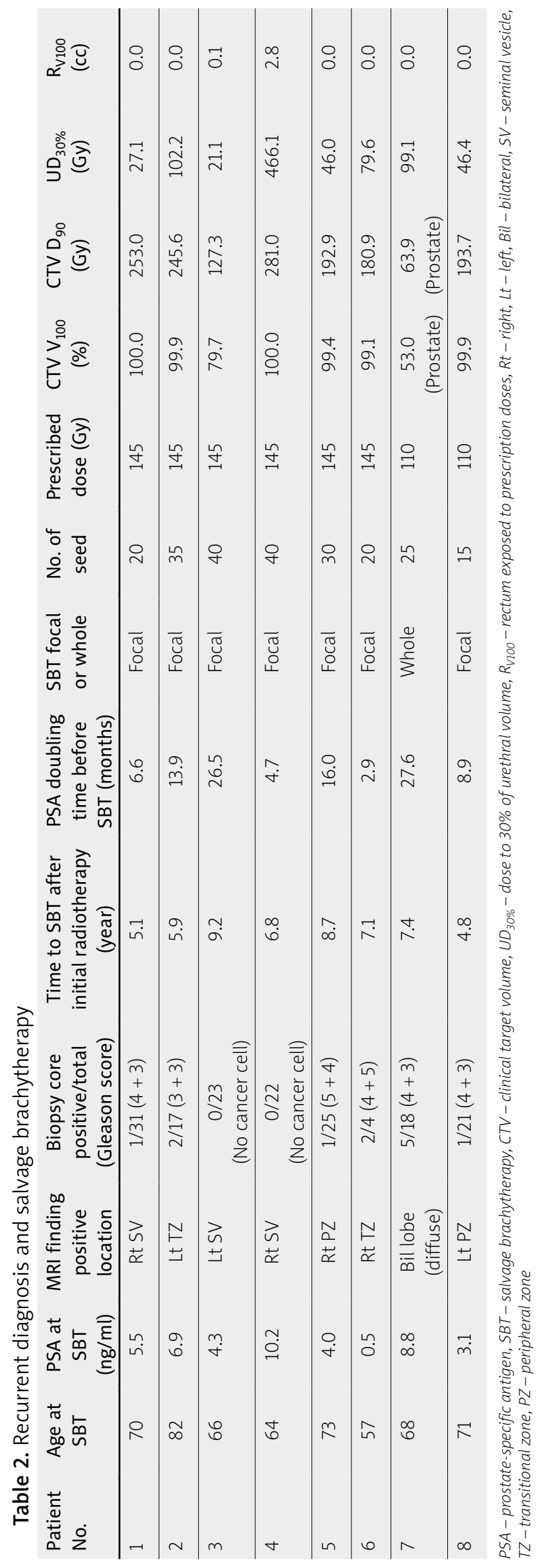

\section{Changes in lower urinary tract symptoms and health-related quality of life}

The pre-SBT baseline (BL) urinary function was prospectively determined by the International Prostate Symptom Score (IPSS) [21] and overactive bladder symptom score (OABSS) [22] before SBT and during the post-SBT follow-ups that were conducted at 1, 3, 6, and 12 months after SBT. The storage symptoms-related IPSS (S-IPSS; the sum of questions 2, 4, and 7) and voiding symptomsrelated IPSS (V-IPSS; the sum of questions 1, 3, 5, and 6) were calculated separately and evaluated [23]. The short form-8 (SF-8) is a self-administered questionnaire that includes an 8-item scale of physical function $(\mathrm{PF})$, role limitation due to physical problems (RP), bodily pain (BP), general health perception (GH), vitality (VT), social function $(\mathrm{SF})$, role limitation due to emotional problems (RE), and mental health (MH) [24]. The SF-8 physical component summary (PCS) and mental component summary (MCS) scores were also calculated from the mean of the scores of items related to physical and emotional health, respectively. The Expanded Prostate Cancer Index Composite (EPIC) measures disease-specific QOL using 10 domains: namely, urinary function, urinary bother, urinary incontinence, urinary irritation/obstruction, bowel function, bowel bother, sexual function, sexual bother, hormonal function, and hormonal bother [25]. Each domain is scaled separately from 0 to 100, with higher scores representing better outcomes. The reliability of the Japanese versions of SF-8, their summary scores, and the EPIC were previously validated in a pilot study carried out in a Japanese population [24,25].

\section{Follow-up after salvage brachytherapy}

Radiation-induced toxicity was graded using the Common Terminology Criteria for Adverse Events (CTCAE), version 4.0. Early and late toxicity were defined as complications occurring within three months post- or pre-SBT, respectively. Toxicity was categorized into GU and GI toxicity. After SBT, patients were evaluated by testing serum PSA every 3 to 6 months for 5 years, and every 12 months thereafter. Disease recurrence after SBT was defined as a PSA increase of $>2 \mathrm{ng} / \mathrm{ml}$ above the PSA nadir level (Phoenix definition [26]) or radiographic tests including MRI.

\section{Statistical analysis}

We evaluated chronological changes by plotting each IPSS, OABSS, and domains of SF-8 and EPIC in line graphs or tables where scores were expressed as the mean \pm standard deviation (SD). The Wilcoxon signed-rank test was used to analyze the changes in variables of LUTS and HR-QOL compared to the baseline. Prism software 7.00 (GraphPad Software, San Diego, CA, USA) was utilized for statistical analyses and data plotting. A $p$ value of $<0.05$ was considered statistically significant.

\section{Results}

\section{Salvage treatment}

Patient characteristics at recurrent diagnosis and SBT are presented in Table 2. The median follow-up pe- 
riod after initial radiotherapy and after LDR-SBT was 8.1 years (range, 5.5-11.0) and 12.2 months (range, 8.3$71.9)$, respectively. The median time between initial radiotherapy and LDR-SBT was 6.9 years (range, 4.89.2). Of the eight cases, six had a biopsy-positive area that corresponded to the positive area from the MRI finding. Of the eight patients, three (patients No. 4, 5, and 6) were treated with neoadjuvant combined ADT for 3-4 months.

A 76-year-old man with $27.0 \mathrm{ng} / \mathrm{ml}$ of initial PSA and GS 6, T2aNOM0 PCa (patient No. 2 in Table 1) had undergone IMRT as a primary radiotherapy. The PSA nadir after initial BT was $1.7 \mathrm{ng} / \mathrm{ml}$. About six years after IMRT, the PSA level was $6.9 \mathrm{ng} / \mathrm{ml}$ and prostate biopsy detected GS 6 lesions in two out of thirty-seven cores from the left transitional zone. With MRI, the recurrent lesion appeared as a high-intensity signal in diffusion-weighted imaging (Figure 1A). Focal SBT was performed with 35 seeds of ${ }^{125} \mathrm{I}$. The PSA level continued to decrease and was low at $0.38 \mathrm{ng} / \mathrm{ml} 18$ months after SBT. A 64-year-old man (patient No. 5 in Table 1) had undergone proton beam therapy as a primary radiotherapy. The PSA nadir after proton beam therapy was $<0.1 \mathrm{ng} / \mathrm{ml}$. About nine years later, the PSA level was $4.0 \mathrm{ng} / \mathrm{ml}$ and prostate biopsy detected GS 9 lesions in one out of 25 cores from the right peripheral zone (Figure 1B). After neoadjuvant ADT for three months, focal SBT was performed with 30 seeds of ${ }^{125} \mathrm{I}$. The PSA level remained low at $0.008 \mathrm{ng} / \mathrm{ml}, 12$ months after SBT.

A

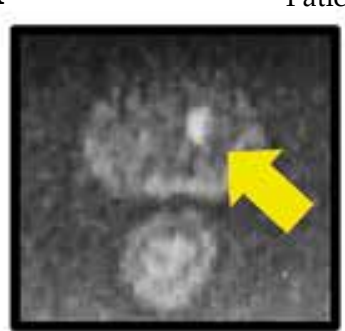

DWI of MRI
Patient No. 2

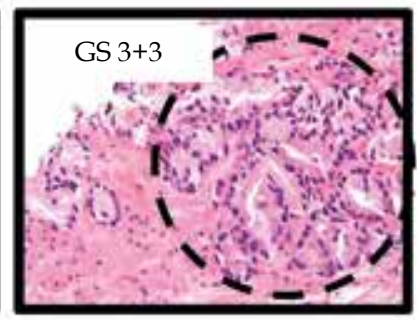

Biopsy H\&E

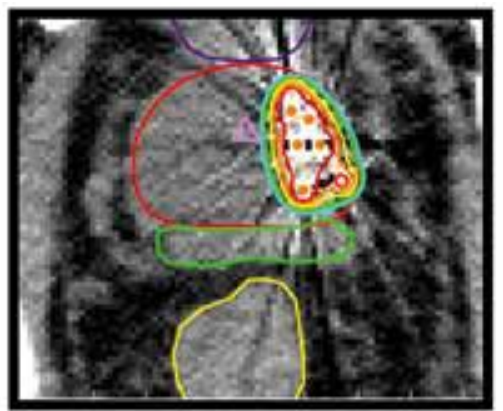

Post-dosimetry on CT
Isodose lines

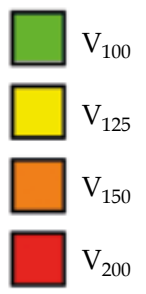

$\mathrm{V}_{200}$

Fig. 1. Two representative cases undergoing salvage brachytherapy. Data of patient No. 2 (A) and No. 5 (B) in Tables 1 and 2 are shown. The left top panel shows an axial diffusion-weighted image (DWI) of magnetic resonance imaging (MRI) before salvage brachytherapy (SBT). The recurrent lesion appears as a high-intensity region (yellow arrow). The right top panel shows H\&E staining of the positive biopsy core. A dashed circle indicates the tumor lesion of Gleason score 3 . The bottom panel shows the post-dosimetry on computed tomography (CT)-imaged axial slices of the prostatic gland after salvage brachytherapy with reimplant isodose distributions for the clinical target volume 

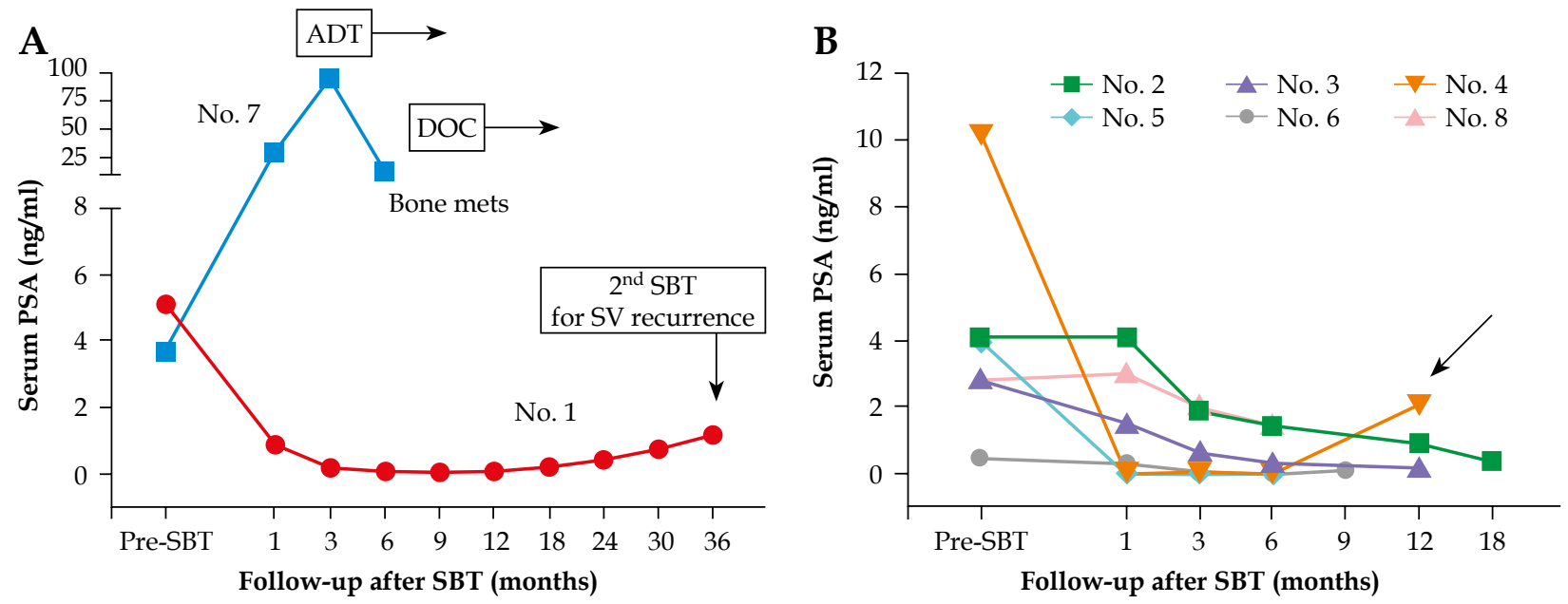

Fig. 2. The longitudinal profile of serum prostate specific antygen (PSA) levels after salvage brachytherapy (SBT) in each patient. A) Two patients experiencing treatment failure. B) Six patients who remained disease-free throughout follow-up. Patient No. 4 was treated with a combination of neoadjuvant androgen deprivation therapy (ADT) and SBT. Serum PSA levels rose from $0.01 \mathrm{ng} / \mathrm{ml}$ to $2.03 \mathrm{ng} / \mathrm{ml}$ at the 12-month follow-up (black arrow). Based on the thought that this is likely due to testosterone recovery or PSA bounce, the patient needs to be closely followed-up. DOC - docetaxel, SV - seminal vesicle

A

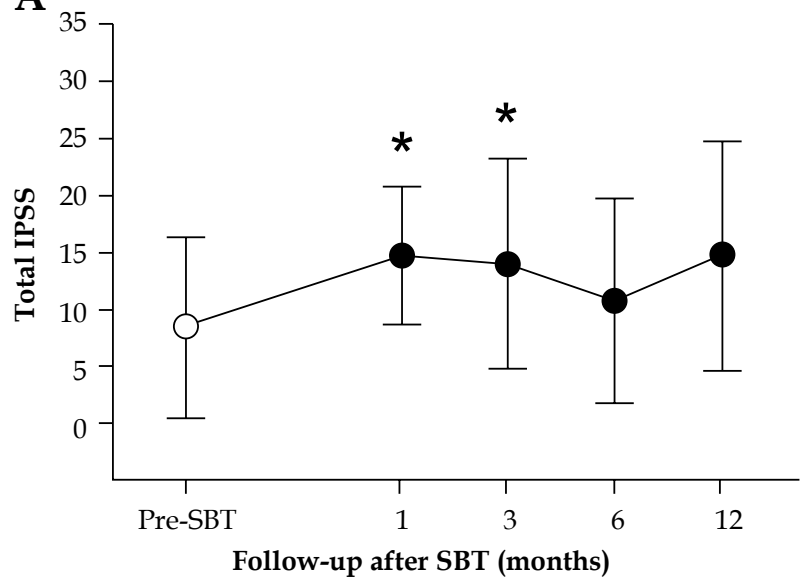

C

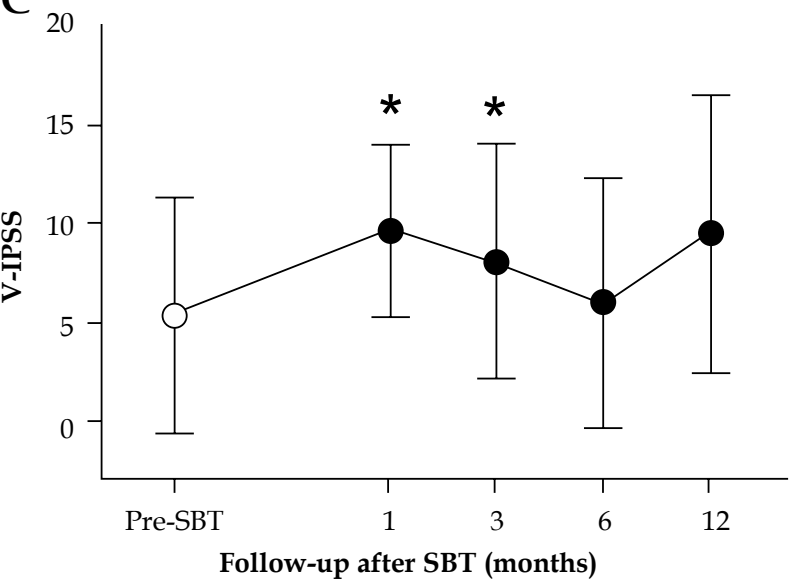

B

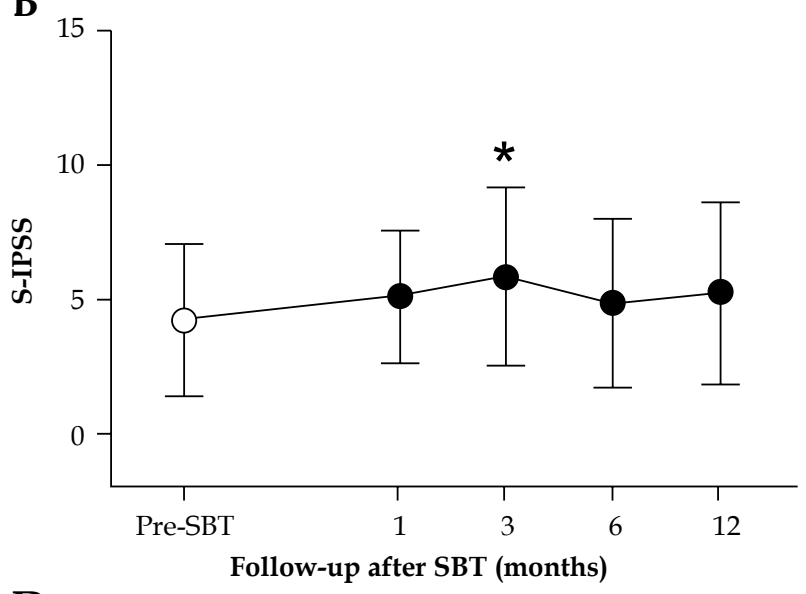

D

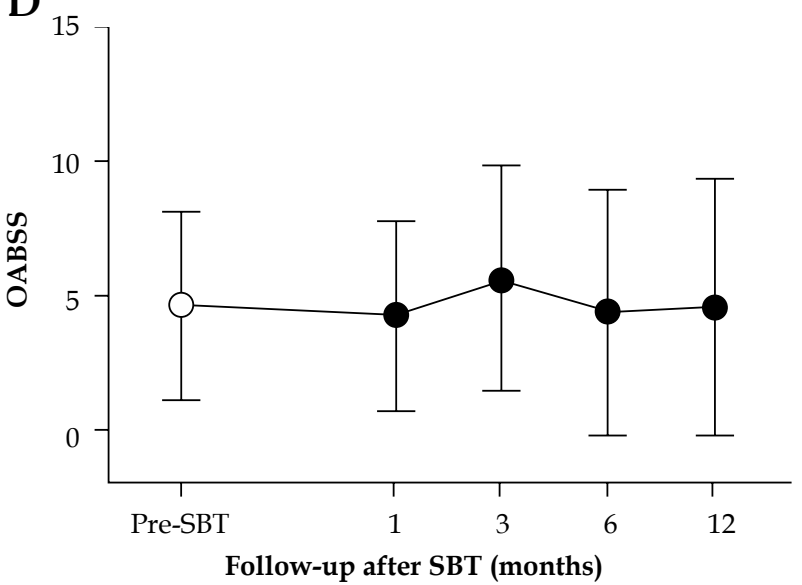

Fig. 3. Changes in parameters for lower urinary tract symptoms (LUTS) during follow-up after salvage brachytherapy (SBT). A) Total score of International Prostate Symptom Score (IPSS). B) The sub score of storage symptoms-related IPSS (S-IPSS); the sum of questions 2, 4, and 7). C) The sub score of voiding symptoms-related IPSS (V-IPSS; the sum of questions 1, 3, 5, and 6). D) The total score of overactive bladder symptom score (OABSS). Data are expressed by means and standard deviations. Scores at each time point $(1,3,6$, and 12 months after implantation) are compared with the baseline scores using the Wilcoxon signed-rank test. ${ }^{*} p<0.05$ 
Table 3. Time-course changes in health-related quality of life using the SF-8 survey

\begin{tabular}{llllll} 
Domains & Baseline & \multicolumn{5}{c}{ Follow-up } \\
\cline { 3 - 5 } & & 1 month & 3 months & 6 months & 12 months \\
\hline Eight scale scores & & & $44.5(11.8)$ & $48.2(9.5)$ & $43.0(17.5)$ \\
\hline PF & $48.1(10.1)$ & $48.5(4.1)$ & $44.7(11.9)$ & $48.4(10.3)$ & $51.9(3.9)$ \\
\hline RP & $49.4(9.8)$ & $48.4(4.6)$ & $53.1(11.0)$ & $56.3(7.0)$ & $60.4(0.0)$ \\
\hline BP & $57.0(4.2)$ & $52.0(8.8)$ & $50.0(9.0)$ & $51.9(7.3)$ & $50.3(0.0)$ \\
\hline VH & $50.7(6.7)$ & $50.0(5.3)$ & $48.8(9.5)$ & $53.3(4.5)$ & $57.9(3.6)$ \\
\hline SF & $54.2(5.2)$ & $47.5(9.4)$ & $42.7(12.1)$ & $46.4(11.2)$ & $55.1(0.0)$ \\
\hline RE & $48.9(10.9)$ & $47.4(7.9)$ & $46.6(8.6)$ & $46.6(8.6)$ & $54.2(0.0)$ \\
\hline MH & $48.4(8.8)$ & $49.9(5.7)$ & $48.4(8.7)$ & $52.6(5.7)$ & $56.9(0.0)$ \\
\hline Summary scores & $47.2(11.6)$ & $51.4(8.1)$ & & & $51.5(2.7)$ \\
\hline PCS & $47.1(6.7)$ & $45.6(6.3)$ & $44.7(9.4)$ & $48.5(6.6)$ & $55.5(1.7)$
\end{tabular}

Standard deviations in parentheses

PF-physical function, RP-role limitation because of physical problems, BP-bodily pain, GH-general health perception, VT-vitality, SF-social function, RE - role limitation because of emotional problems, MH - mental health, PCS - physical component summary, PCS - mental component summary, SF-8 - medical outcomes study 8-item short form

Table 4. Time-course changes in health-related quality of life using the Expanded Prostate Cancer Index Composite (EPIC)

\begin{tabular}{llllll} 
Domains & Baseline & \multicolumn{3}{c}{ Follow-up } \\
\cline { 3 - 5 } & & 1 month & 3 months & 6 months & 12 months \\
\hline Urinary & $91.5(11.9)$ & $88.5(16.0)$ & $85.5(19.4)$ & $88.4(14.2)$ & $84.5(26.9)$ \\
\hline Function & $95.3(12.5)$ & $92.4(15.9)$ & $85.3(24.2)$ & $93.6(14.9)$ & $84.4(27.0)$ \\
\hline Bother & $88.8(12.9)$ & $85.7(16.8)$ & $85.7(17.9)$ & $84.7(15.7)$ & $84.5(26.8)$ \\
\hline Irritation/Obstruction & $89.6(22.5)$ & $87.8(23.2)$ & $85.1(29.2)$ & $86.6(26.1)$ & $74.3(44.6)$ \\
\hline Incontinence & $95.9(5.6)$ & $92.3(11.4)$ & $89.8(15.1)$ & $92.9(8.5)$ & $92.9(12.4)$ \\
\hline Bowel & $90.1(13.6)$ & $93.9(2.7)$ & $96.4(2.9)$ & $96.2(3.2)$ & $100(0.0)$ \\
\hline Function & $93.9(4.5)$ & $90.3(5.3)$ & $95.9(4.3)$ & $93.9(5.3)$ & $100(0.0)$ \\
\hline Bother & $93.4(7.0)$ & $97.4(2.7)$ & $96.9(3.8)$ & $98.5(2.8)$ & $100(0.0)$ \\
\hline Sexual & $32.1(12.8)$ & $32.6(2.3)$ & $35.7(7.9)$ & $30.8(13.1)$ & $28.6(21.1)$ \\
\hline Function & $12.2(10.7)$ & $5.3(7.0)$ & $8.3(14.3)$ & $7.1(15.5)$ & $13.6(21.2)$ \\
\hline Bother & $76.8(33.2)$ & $92.9(18.9)$ & $97.3(7.1)$ & $83.9(31.0)$ & $62.5(41.0)$ \\
\hline Hormonal & $94.8(7.0)$ & $94.2(7.4)$ & $89.6(11.1)$ & $92.9(8.5)$ & $97.0(2.6)$ \\
\hline Function & $92.1(10.7)$ & $88.6(13.1)$ & $83.6(13.8)$ & $88.6(11.8)$ & $93.3(5.8)$ \\
\hline Bother & $97.0(5.2)$ & $98.8(3.1)$ & $94.6(9.2)$ & $96.4(6.1)$ & $100(0.0)$
\end{tabular}

Standard deviations in parentheses

For total IPSS and V-IPSS, the lowest symptom scores were observed 1 month after implantation; however, the scores decreased with time (Figures 3A and C). Although the V-IPSS showed significant increases at 1 and 3 months, the S-IPSS only showed a significant increase at 3 months (Figure 3B). No significant change was seen in the OABSS (Figure 3D). These findings support the idea that transient deterioration in LUTS after the SBT is attributed to voiding symptoms rather than storage symptoms. 
Changes in the scores for the eight domains and the two summary scores of the SF- 8 survey and 10 domains of the EPIC are summarized in Tables 3 and 4, respectively. The scores of all domains remained unchanged during follow-up after SBT.

\section{Salvage brachytherapy-induced toxicity}

The incidence of early and late toxicity during the LDR-SBT and first 12 months after SBT are shown in Figure 4. Early grade 1 and 2 GU toxicity (including pollakisuria, urgency, and incontinence) was observed in four $(50 \%)$ and one (13\%) patients, while late grade 1 and 2 GU toxicity was observed in two (25\%) and one (13\%) patients, respectively. No early GI toxicity was observed, whereas grade 2 GI toxicity was observed in one patient (13\%) who had persistent diarrhea. No patient required intermittent catheterization and no grade 3 or greater toxicity occurred during follow-up in the cohort.

\section{Discussion}

A previous survey investigating the treatment distribution of primary therapy for CT1-2N0M0 PCa at our institute showed a radiotherapy rate of $45 \%$ [7]. We previously reported that the PSA failure-free rate using the Phoenix definition in patients treated with ${ }^{125} \mathrm{I}$ seed implantation was $92.8 \%$ at 5 years [27]. In general, up to $10-15 \%$ of patients may experience PSA failure in $5-10$ years after seed implantation, which requires salvage treatments [28]. Post-treatment sensitive monitoring with PSA, ultrasound-guided prostate re-biopsy, and recent advancements in imaging technologies enables the early detection of local recurrent tumors after primary radiotherapy. The NCCN version 2017 guideline currently endorses salvage local therapy consisting of salvage prostatectomy, SBT, or salvage cryotherapy as reasonable alternatives to observation or ADT for selected patients with a biopsy-proven local recurrence after primary radiotherapy for localized PCa [5].

The selection of salvage modality involves weighing the oncological effectiveness and treatment-induced toxicity of each treatment. Parekh et al. reported a systematic

A

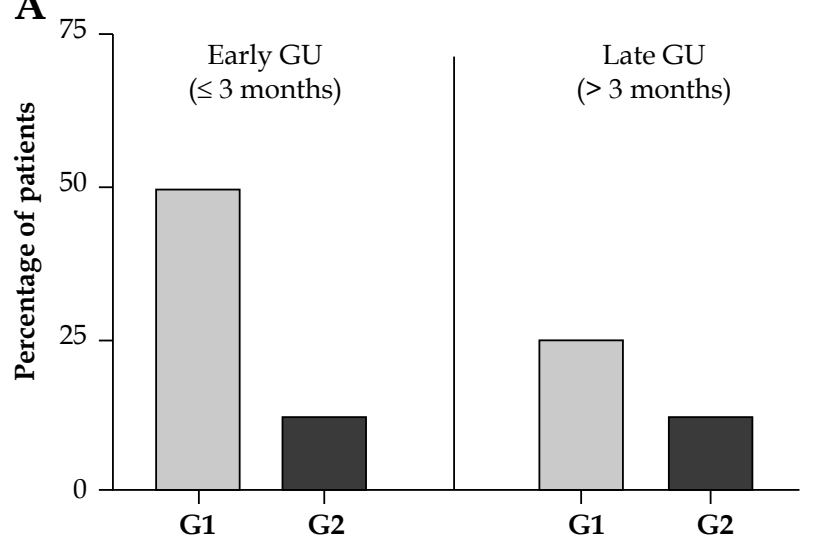

review of the literature on the oncologic outcomes and toxicity of four salvage local therapies consisting of BT, prostatectomy, cryotherapy, and HIFU [3]. The review stated that 5-year failure-free survival (FFS) rates between these were similar, ranging from $52 \%$ to $57 \%$. Because of the differences in patient selection and definition of biochemical failure in the comparison of previous reports, we could not conclude the superiority or inferiority between the salvage modalities. The publications from the Mayo Clinic series (2006), the Dana Farber series (2007), and the Mount Sinai series (2010) reported a 5-year FFS rate of $75 \%$ at 4 years using the American Society for Radiation Oncology (ASTRO) definition [9], 70\% using the Phoenix definition [10], and 65\% using the Phoenix definition [11], respectively. To date, the largest series of LDR-SBT is that published from Vargas et al. in 2014 and includes 69 patients with a 5-year FFS rate of $73.8 \%$ for no-castration resistant PCa patients [15]. As to our series, the sample size was small and the follow-up period was short. However, treatment failure was observed in only $25 \%$ of patients in our series, which seems to be acceptable. Patient No. 7 in Table 1 developed rapid elevation of PSA without any decline after LDR-SBT and was diagnosed with multiple bone metastases (Figure 2A). The experience of this case emphasizes that detecting the sites of failure is vital for selecting the appropriate salvage modality. Identification of candidates is based on clinicopathologic variables including post-treatment PSA kinetics, such as PSADT [29]. D'Amico et al. demonstrated that the optimal candidate for local salvage therapy includes more than 3 years of PSA failure, 12 months or more of post-treatment PSADT, a Gleason score of less than 8 in biopsy specimens, and no SV involvement [29]. Long-term observation after SBT published by Burry et al. demonstrated that pre-salvage PSA levels $<6 \mathrm{ng} / \mathrm{ml}$ were independently associated with better FFS [11]. Of two recurrent cases in our series, patient No. 1 had SV involvement and short PSADT (6.6 months), among known poor factors. In patient No. 7, the recurrent tumors did not seem to involve local regions, but involved radiographically undetectable metastases of the bone (Figure 2A). The PSADT was 27.6 months and the Gleason score in biopsy specimens was $4+3$; however, this patient had high pre-salvage PSA levels $(8.8 \mathrm{ng} / \mathrm{ml}$,

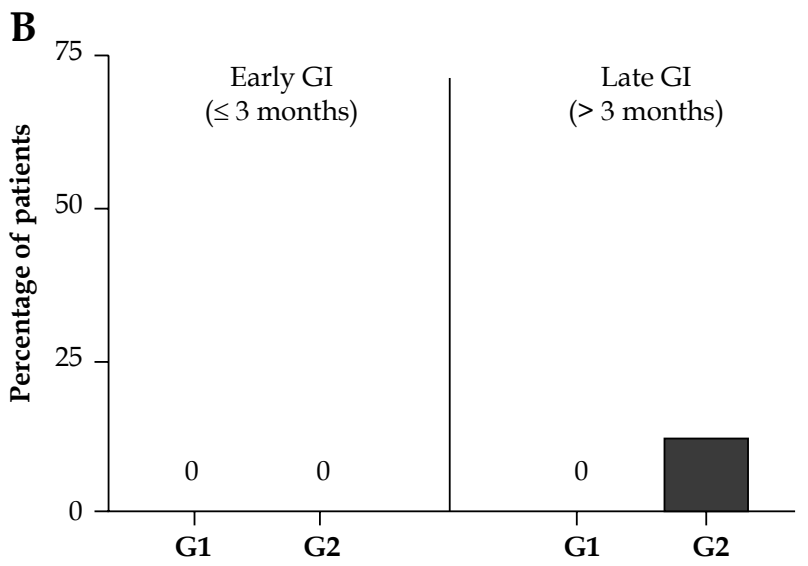

Fig. 4. Early and late toxicity after SBT. Early and late toxicity was defined as complications occurring within 3 months pre- and post-SBT, respectively. Toxicity was categorized into genitourinary (GU) and gastrointestinal (GI) toxicity 
Table 2). We expected that LDR-BT could eradicate the recurrent tumor because the patient exhibited only one risk factor among known poor factors. More accurate risk prediction tools are required to avoid unnecessary local salvage therapy and initiate ADT as early as possible.

We are cautious when pre-planning and seeding to minimize the exposure dose to the urethra and rectal wall. In our series, no patient required urinary catheterization and no grade 3 or greater toxicity occurred during follow-up. The systematic review of 13 studies by Parekh et al. reported that the rates of toxicity after LDR-BT were $12.9 \%$ for grade $3-4$ GU toxicity and $4.7 \%$ for grade $3-4$ GI toxicity. The GU toxicity included urethral strictures and urinary incontinence. The GI toxicity included $3.1 \%$ for recto-urinary fistulas and $7.5 \%$ for stricture. There was wide variability across the series. For instance, Nguyen et al. used an MRI-guided SBT with ${ }^{125} \mathrm{I}$ implantation and reported that the rates of grade 3-4 GI or GU was 30\% during the 47 -month median follow-up, in which $13 \%$ of patients required a colostomy and/or urostomy to repair a recto-urinary fistula. An observation after HDR-SBT showed that the biologically effective dose 2 Gy $(\alpha / \beta 1.5 \mathrm{~Gy})$ levels $\geq 227$ and ADT were significant predictors of grade 2 or greater GU toxicity [12].

Another major concern of LDR-SBT is the change in QOL. Information regarding associated post-SBT deterioration in LUTS and HR-QOL should be provided to patients prior to the salvage treatment to help patients make informed decisions. We previously reported changes in LUTS and HR-QOL after salvage radiotherapy for biochemical recurrence in patients undergoing radical prostatectomy [23]. To date, there have been few studies investigating changes in LUTS after LDR-SBT [12]. To our knowledge, no study evaluating chronologic changes in HR-QOL after LDR-SBT has been published. We found a significantly raised total IPSS that remained consistent for 1-3 months after LDR-SBT, after which it returned to baseline. This result is similar with that reported by Rose et al. [12]. The greater deterioration in voiding symptoms than in storage symptoms was responsible for the greater total IPSS seen in our study. An overall stability in general HR-QOL (SF-8) and disease-specific QOL (EPIC) was observed in patients treated with LDR-SBT in our study. In our assessment of EPIC, we identified no significant decline in urinary, bowel, hormone, or sexual function and bother. The EPIC questionnaire includes incontinence-specific parameters such as the incontinence score. The number of incontinence pads used per day might be another useful parameter for measuring sequential changes in incontinence.

Limitations of this study include the small sample size, which lowers the ability to obtain significant results. This was a single-institution non-randomized study. Moreover, assessment with the IPSS, OABSS, SF-8, and EPIC questionnaires was not frequently after LDR-SBT. More frequent assessments, such as once every month or 2 months, may improve the findings.

\section{Conclusions}

To our knowledge, this is the first study to evaluate LUTS, HR-QOL, and disease-specific QOL after LDR-SBT at specific time-points during and early in the post-treatment period in PCa patients. We believe that this study has provided important insights concerning time course changes in LUTS, HR-QOL, and disease-specific QOL for patients receiving LDR-SBT. A better understanding of the QOL outcomes associated with this modality may enable patients to make better-informed decisions regarding treatment for recurrent PCa.

\section{Acknowledgments}

The authors are grateful to the patients who consented to the usage of the clinical information.

\section{Disclosure}

Authors report no conflict of interest.

\section{References}

1. Sharifi N, Gulley JL, Dahut WL. Androgen deprivation therapy for prostate cancer. JAMA 2005; 294: 238-244.

2. Zdrojowy R, Dembowski J, Małkiewicz B et al. Salvage local therapy for radiation-recurrent prostate cancer - where are we? Cent European J Urol 2016; 69: 264-270.

3. Parekh A, Graham PL, Nguyen PL. Cancer control and complications of salvage local therapy after failure of radiotherapy for prostate cancer: a systematic review. Semin Radiat Oncol 2013; 23: 222-234.

4. Duijzentkunst DA, Peters M, van der Voort van Zyp JR et al. Focal salvage therapy for local prostate cancer recurrences after primary radiotherapy: a comprehensive review. World J Urol 2016; 34: 1521-1531.

5. Mohler JL, Armstrong AJ, Bahnson RR et al. Prostate Cancer, Version 1.2016. J Natl Compr Canc Netw 2016; 14: 19-30.

6. Crouzet S, Murat FJ, Pommier P et al. Locally recurrent prostate cancer after initial radiation therapy: early salvage high-intensity focused ultrasound improves oncologic outcomes. Radiother Oncol 2012; 105: 198-202.

7. Tanaka N, Fujimoto K, Hirayama A et al. The primary therapy chosen for patients with localized prostate cancer between the university hospital and its affiliated hospitals in Nara Uro-Oncological Research Group registration. BMC Urol 2011; 11: 6.

8. Grado GL, Collins JM, Kriegshauser JS et al. Salvage brachytherapy for localized prostate cancer after radiotherapy failure. Urology 1999; 53: 2-10.

9. Wong WW, Buskirk SJ, Schild SE et al. Combined prostate brachytherapy and short-term androgen deprivation therapy as salvage therapy for locally recurrent prostate cancer after external beam irradiation. J Urol 2006; 176: 2020-2024.

10. Nguyen PL, Chen MH, D'Amico AV et al. Magnetic resonance image-guided salvage brachytherapy after radiation in select men who initially presented with favorable-risk prostate cancer: a prospective phase 2 study. Cancer 2007; 110: 1485-1492.

11. Burri RJ, Stone NN, Unger P et al. Long-term outcome and toxicity of salvage brachytherapy for local failure after initial radiotherapy for prostate cancer. Int J Radiat Oncol Biol Phys 2010; 77: 1338-1344.

12. Henríquez I, Sancho G, Hervás A et al. Salvage brachytherapy in prostate local recurrence after radiation therapy: predicting factors for control and toxicity. Radiat Oncol 2014; 9: 102.

13. Sasaki H, Kido M, Miki K et al. Salvage partial brachytherapy for prostate cancer recurrence after primary brachytherapy. Int J Urol 2014; 21: 572-577. 
14. Vargas C, Swartz D, Vashi A et al. Salvage brachytherapy for recurrent prostate cancer. Brachytherapy 2014; 13: 53-58.

15. Rose JN, Crook JM, Pickles T et al. Salvage low-dose-rate permanent seed brachytherapy for locally recurrent prostate cancer: Association between dose and late toxicity. Brachytherapy 2015; 14: 342-349.

16. Kunogi $\mathrm{H}$, Wakumoto $\mathrm{Y}$, Yamaguchi $\mathrm{N}$ et al. Focal partial salvage low-dose-rate brachytherapy for local recurrent prostate cancer after permanent prostate brachytherapy with a review of the literature. J Contemp Brachytherapy 2016; 8: 165-172.

17. D'Amico AV, Whittington R, Malkowicz SB et al. Biochemical outcome after radical prostatectomy, external beam radiation therapy, or interstitial radiation therapy for clinically localized prostate cancer. JAMA 1998; 280: 969-974.

18. Miyake M, Tanaka N, Asakawa I et al. Proposed salvage treatment strategy for biochemical failure after radical prostatectomy in patients with prostate cancer: a retrospective study. Radiat Oncol 2014; 9: 208.

19. Tanaka N, Asakawa I, Kondo $\mathrm{H}$ et al. Technical acquisition and dosimetric assessment of iodine-125 permanent brachytherapy in localized prostate cancer: our first series of 100 patients. Int J Urol 2009; 16: 70-74.

20. Tanaka N, Torimoto K, Asakawa I et al. Use of alpha- 1 adrenoceptor antagonists in patients who underwent low-doserate brachytherapy for prostate cancer - a randomized controlled trial of silodosin versus naftopidil. Radiat Oncol 2014; 9: 302.

21. Homma Y, Kawabe K, Tsukamoto T et al. Epidemiologic survey of lower urinary tract symptoms in Asia and Australia using the International Prostate Symptom Score. Int J Urol 1997; 4: 40-46.

22. Homma Y, Kakizaki H, Yamaguchi O et al. Assessment of overactive bladder symptoms: comparison of 3-day bladder diary and the overactive bladder symptoms score. Urology 2011; 77: 60-64.

23. Miyake M, Tanaka N, Asakawa I et al. Changes in lower urinary tract symptoms and quality of life after salvage radiotherapy for biochemical recurrence of prostate cancer. Radiother Oncol 2015; 115: 321-326.

24. Sugimoto M, Takegami M, Suzukamo Y et al. Health-related quality of life in Japanese men with localized prostate cancer: assessment with the SF-8. Int J Urol 2008; 15: 524-528.

25. Kakehi Y, Takegami M, Suzukamo Y et al. Health related quality of life in Japanese men with localized prostate cancer treated with current multiple modalities assessed by a newly developed Japanese version of the Expanded Prostate Cancer Index Composite. J Urol 2007; 177: 1856-1861.

26. Roach M 3rd, Hanks G, Thames H Jr et al. Defining biochemical failure following radio therapy with clinically localized prostate cancer: recommendations of the RTOG-ASTRO Phoenix Consensus Conference. Int J Radiation Oncol Biol Phys 2006; 65: 965-974.

27. Tanaka N, Asakawa I, Katayama E et al. The biochemical recurrence-free rate in patients who underwent prostate lowdose-rate brachytherapy, using two different definitions. Radiat Oncol 2014; 9: 107.

28. Lacy JM, Wilson WA, Bole R et al. Salvage Brachytherapy for Biochemically Recurrent Prostate Cancer following Primary Brachytherapy. Prostate Cancer 2016; 2016: 9561494.

29. Lee AK, D'Amico AV. Utility of prostate-specific antigen kinetics in addition to clinical factors in the selection of patients for salvage local therapy. J Clin Oncol 2005; 23: 8192-8197. 